\title{
Unsupervised Learning of a Rule-based Spanish Part of Speech Tagger
}

\author{
Chinatsu Aone and Kevin Hausman \\ Systems Research and $\Lambda$ pplications Corporation (SR $\Lambda$ ) \\ 4300 Fair Lakes Gourt \\ lairlax, V^22033 \\ aonec(osta.com, hausmandosra.com
}

\begin{abstract}
'This paper describes a Spamish Part-ol'. speech (POS) lagger which applies and extends Brill's algorithom for unsupervised learning of rule-based taggers (Brill, 1995). First, we discuss our general approach including extensions we made tio the algorithru in order to handle unknown words and paramederize Iearning and tagging options. Next, we report and analyo ont experimental pesultis us. ing diflorent paraneters. 'Then, wo de scribe our "hybricl" approach which was necessary in order to overeomre a Ciundamontal limitation in Brill's original algorithun. Finally, we compare our hayger with Ilidelen Markov Model (IIMM)based taggers.
\end{abstract}

\section{Introduction}

We have developed a Spanish Pari-eospech (POS) 'lagger which applies and extends Brill's algorithm for unsupervised learuing (Brill, 1995) to creatie a sed of rules that reduce the ambiguty of Pos tagis on words. Wo have chosen an unsupervised learning algorithm becanse it does not require a large Pos-tagged traning corpus. Since there was no POS tagged Spanish corpuls available to us and since creating a large hand-tagged coppus is both costly and prone to inconsistency, the decision was also a practical one. Wo have decieled to develop a rule-based tagger because sucts a taggerer learns a set of declarative rules and also becanse we wanted to compare it with llidden Markov Model (IIMM)-bassed taggers.

We extended Brill's algorithm in several ways. l'irst, we extended it to handle unknown words in the training and test texts. Socond, we paraneterized learning and tagging options. Finally, we experimented with a "hybrid" solution, where we used a very small number of hand-elisambiguated texts during training to overeome a fundamental limitation in the learning algorithun.

\section{Components}

Our Spanish Pos tagger consistis of three comper nents: the Initial Stater Annotator, the Teamer and the Rule 'lagger, each of which is described below.

\subsection{Initial State Annotator}

'This component is used to assign all possible POS tages to a given Spanist word. It consists of lexicon lookup, momphological analysis, and un known word handling. 'I'te Spanisth Pos tag set. used in this work comsists of the following tags

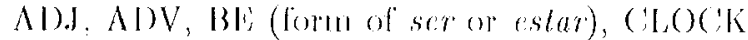

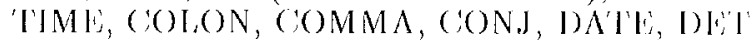
IIAVli (form of haber), IIYPIIEN, LI,IOITR,

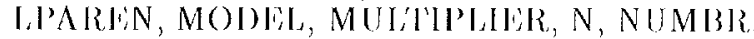

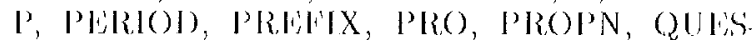
MARK, QUOTR, ROMAN, RIPAREN, SHMJ GOLON, SLASH, SUBCON.J, SULPIX, THLRE (hacer used in "ihere" constructions), WHD I (cuál libro), WIINP' (quo), WIIPP' (dónde), and V (sece l'ablo :3).

\subsubsection{Lexicon Lookup and Morphological Analysis}

Unlike Brill's linglish tagger experiment described in (Brill, 1995), no large Pos-bagged Spanish corpus was available to 12 from which a large lexicon can bo derived. As a result, we de cided to palse the on-line Collins Spanish-linglish Dictionary', and derived a large lexicon from it (about 15000 entries). We used only the opentclass entries from this lexicon, and then any mented it, with irregulas verb forms and a number of closed-class words. Our morphological analyzer uses a sed of rewrite rules to strip off and/or mod ify word curlings to find root forms of words.

\subsubsection{Jnknown Word Handling}

Since the lexicon and tuorphological analysio will not cover every single word that can appear in a text, an attempt is made at this stage to classify unknown words. Any word which rlid not get assigned one or nore parts-ol-speceh in

\footnotetext{
${ }^{1}$ We have obtained a license to the dictionary.
} 
the lookup/morphology phase is examined for certain traits often indicative of particular parts-of specch. This task is similar to what was done by the guessers for the HMM-based French and German taggers (Chanod and Tapanainen, 1995; Feldweg, 1995).

For example, words ending in the letters "mente" are assigned the tag of ADV (adverb). Those words ending in "ando" or "endo" are assigned the tag V-CONTINUOUS-NIL (continuous form of the verb). Table 1 shows a list of unknown word handling rules.

Table 1: Unknown Word Handling Rules

\begin{tabular}{|l|l|}
\hline Heuristics & POS tag \\
\hline num $>1600 \&<2100$ & DATE \\
roman numeral 1-9 & ROMAN \\
-and,-endo & V-CONTINUOUS-NIL, \\
-ido,-ado,-ida,-ada & V-PERFECT-NIL \\
-er,-ir,-ar & V-NI,-NIL \\
-erse,-irse,-arse & V-NII-NIL-CLITIC \\
-ción,-idad,-izaje & N \\
-mente & ADV \\
-able & ADJ \\
capitalized & PROPN \\
\hline
\end{tabular}

Performing these simple checks reduces the number of unknowns in our test set of 17,639 words from $737(4.2 \%)$ to $158(0.9 \%)$. The remaining unknowns are assigned a set of ambiguous open-class tags of $\mathrm{N}, \mathrm{V}, \mathrm{ADJ}$, and ADV so that they can be disambiguated by the Learner.

\subsection{Learner}

The Learner takes as input ambiguously tagged texts produced by the Initial State Annotator, and tries to learn a set of rules that will reduce the arrbiguity of the tags. Output is a file of rules in the following form:

$$
\begin{gathered}
\text { context }=C: P_{1}|\ldots| P_{i}|\ldots| P_{n} \rightarrow P_{i}, \text { where } \\
\text { context is one of PREVWORD, } \\
\text { NEXTWORD, PREVTAG or NEXTTAG, } \\
C \text { is a word or tag, } \\
P_{1}, \ldots, P_{i}, \ldots, P_{n} \text { are the ambiguous } \\
\text { parts-of-speech to bo reduced, } \\
P_{i} \text { is the part-of-speech that replaces } \\
P_{1}, \ldots, P_{i}, \ldots, P_{n} \text {. }
\end{gathered}
$$

Here are some examples taken from the actual learned rules:

- $\operatorname{NEXTWORD}=\mathrm{DE}: \mathrm{P} \mid \mathrm{N} \rightarrow \mathrm{N}$

- PREVWORD = EN : DET $\mid$ ADV $\rightarrow$ DET

- PREVTAG = DET $: \mathrm{V} \mid \mathrm{N} \rightarrow \mathrm{N}$

- $\mathrm{NEXTTAG}=\mathrm{SUBCONJ}: \mathrm{BE} \mid \mathrm{V} \rightarrow \mathrm{V}$

\footnotetext{
${ }^{2}$ PREVWORD $=$ previous word, PREVTAG $=$ previous tag.
}

The Learner applies Brill's algorithm for unsupervised learning to try to reduce the ambiguity of the tags in the input corpus. 'The following steps are taken:

1. The Learner examines each ambiguously tagged word and creates a set of contexts for the word. Two of these contexts will be PREVWORD and NEXIWORD. The remainder consist of PREV'IAG and NEXTTAG contexts as required by the tag(s) on the preceding and following words. For example, if the word preceding the ambiguously tagged word is ambiguously tagged with two tags, then the Learner must generate two PREV'TAG contexts.

2. An attempt is made to find unambiguously tagged words in the corpus that are tagged with one and only one of the tags on the ambiguously tagged word. For example, if the word in question has both $\mathrm{N}$ and $V$ tags, then the Iearner would search for words with only an $N$ tag or only a $V$ tag.

3. If such a word is found, the contexts of that word are examined to determine if there is an overlap between them and the contexts generated for the ambiguously tagged word. One issue for this determination is how much ambiguity should be tolerated in the context of the unambiguously tagged word. For example, if one of the possible contexts is PRli$V T A G=N$ and the word preceding the unambiguously tagged word has both $\mathrm{N}$ and $\mathrm{V}$ tags, should the context apply? To permit various approaches to be tried, we extended the Learner to accept a parameter (i.e., freedom) that determines how much ambiguity will be accopted on the context words for the context to match.

4. If a context matches for this unambiguously tagged word, the count of unambiguously tagged words with the particular part of speech occurring in that context is incremented.

5. After the entire corpus is examined, each of these possible reduction rules (of the form "Change the tag of a word from $\chi$ to $Y$ in the context $\mathrm{C}$ where $\mathrm{Y} \in \chi^{\prime \prime}$ ) is ranked according to the following. First, for each tag $\mathrm{Z} \in \chi, \mathrm{Z} \neq \mathrm{Y}$, the Learner computes:

$$
\frac{\text { fre } q(Y)}{\text { freq( }(Z)} * \text { incontext }(Z, C), \text { where }
$$

freq $(Y)=$ number of occurrences of words unambiguously tagged with $\mathrm{Y}$,

freq $(Z)=$ number of occurrences of words unambiguously tagged with $Z$,

incontext $(Z, C)=$ number of times a word unambiguously tagged with $\mathrm{Z}$ occurs in context, $\mathrm{C}$. 
'The tag $\%$ that gives the highest score from this formula is saved as R. 'Then the score for a particular transformation is

$$
\text { incontext }(Y, C)-\frac{f r e q(Y)}{f r e q(R)} * i n c o n t c x t(R, C)
$$

6. If the highest-ranked transformation is not positive, the Learner is done. Otherwise, the highest-ranked transformation is appended to the list of transformations learned. 'The Learner then searches this list for the transformation that will result in the most reduction of ambiguity (which will always be the latest rule learned) and applies it. 'This process continues until no further reduction of ambiguity is possible. Here, we also extended the Learner to accept a different paraneter (i.c., l-tagfreedom) that determines how much ambiguity will be accepted on a word that is used for context, during ambiguily reduction, that is, when the Learner has found a rule and is applying it to the training text. Note that specifying too small a value for this parameter can cause the learner to go into an endless loop, as restricting the valid contexts may have the effect of nullifying the just-learned rule.

7. 'l'he Iearner then returns to step 1 to begin the process again

\subsection{Rule Tagrox}

This component reads tagged texts produced by the Initial State Annotator and rules produced by the Learner and applies the learned rules to the tagged texts to reduce the ambiguity of the tags.

We extended the Rule 'Iagger to have two possible modes of operation (i.e., best-rule-first and learned-sequence modes controlled by the seq parameter) for using the learned rules to reduce anbiguity:

1. The Rule 'lagger can use an algorithm similar to that used in step 7 of the Icamer. Wach possible reduction rule is examined against the text to determine which rule results in the greatest reduction of ambiguity.

2. The Rule Tagger can use a sequential application of the learned rules in the order that the rules were learned. After each rule has been applied in sequence, all of the rules preceding it are re-applied to take advantage of ambiguity reductions made by the latest. rule: applied.

The Rule Tagger allows one to specily, as in the Learner, how much ambiguity will be tolerated for a context to match. lior example, one can be very restrictive and require that a tag context (e.g., PREV'lAG $\mathrm{N}=\mathrm{N}$ ) match only an unambiguousily tagged word (in this case, a word with only an $\mathrm{N}$ tag). 'Ihis parameter (i.e., r-lagfreedom) specifies the maximum ambiguity allowed on a context, word for a context tag to match: I requires that the context word be unambiguously tagged, 2 requires that there be no more than two tatgs on the word, and so on.

\section{Experiments and Results}

for training and testing of the tagger, we have raudomly picked articles from a large (274MB) "Hol Norte" Mexican newspaper corpus, and sej)arated then into the training and test sets. The test set $(17,639$ words) was lagged manually for comparisou against the system-tagged texts. For training, we partitioned the development set into several dilferent-sized sets in order to see the of fects of training corpus sizes. 'Tho broakdown call be found in 'Table 2

'Table 2: Ambignously lagged 'Training sets

\begin{tabular}{|l|l|}
\hline Sot & Words \\
\hline 'Tiny & 1322 words \\
Simall & 3066 words \\
Medium & 5591 words \\
Full & 12795 words \\
\hline
\end{tabular}

If one randomly picks one of the possible tags on ach word in the test set, the accuracy is $78.0 \%$ (78.6\% with the simple verb tag set). The average los ambiguily per word is $1.52(1.49)$ inchurling punctuation lags and $1.58(1.56)$ excholing punctuation lags. lor comparison, the accuracy of Brill's unsupervised bnglish tagger was $95.1 \%$ using 120,000-word P'enn Treebank texts. Ilis initial state tagging accuracy was $90.7 \%$, which is considerably higher than our Spanish case (78.6\%).

\subsection{Effect of Tay Sot}

Our first set of experiments tests the effect of the Pos tag complexity. We used both the simple verb tag set (5 tags) and the complex verb tag set (42 tags), which is shown in Table 3, where * can be cither $1 S G, 2 S(x, 3 S G, 1 P L, 2 P I$, or 3PI. In the case of simple verb tag set, tense, person and number information is discarded, leaving only at "V" tag and the lower four tags in the table.

The scores with the simple verb tag set for dif. ferent sizes of training sets are found in Table 4, and those with the complex verb tag set in 'l'able 5. For these two experinents, the Learner was sot to have a tight restriction on using context for learning (i.e, the freedom parameter was set to 1) and a loose restriction on context for applying the learned rules (i.c., l-tagfreedom 10). The Rule 'Tagger was given a moderately-tight restriction on using context for reduction rule application (i.e., $r$-tagfreedom 2).

In general, the scores are slightly higher using the simple verb tag set over the complex verb 
Table 3: Complex Verb 'I'ag Sot

\begin{tabular}{|c|}
\hline 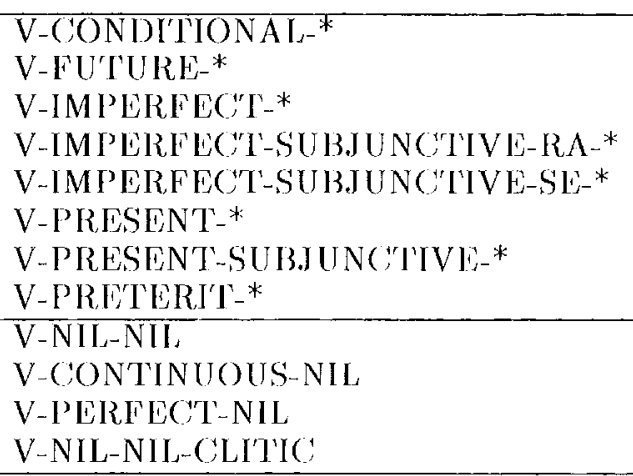 \\
\hline
\end{tabular}

Table 4: Ambiguously tagged texts, Simple Verbs

\begin{tabular}{|l|c|c|}
\hline Set & \# of rules learned & Score \\
\hline T'iny & 131 & $82.5 \%$ \\
Small & 211 & $91.5 \%$ \\
Medium & 287 & $91.8 \%$ \\
l'ull & 134 & $83.0 \%$ \\
\hline (none) & 0 & $78.6 \%$ \\
\hline
\end{tabular}

tag set $(91.8 \%$ vs. $90.3 \%$ for the "Medium" corpus). This behavior is most likely due to the fact that some verb tense/person/number combinations cannot easily be clistinguished from context, so the Learner was unable to find a rule that would disambiguate them.

As can be seen from the tables, performanco increased as the size of the learning set increased up to the "Medium" set, where the score levelled off. With very small learning sets, the system was unable to find sufficient examples of phenomena to produce reduction rules with good coverage.

One surprising data point in the simple verb tag set experiments was the "Full" score, which dropped almost 9\% from the "Medium" score. After analyzing the results more closely, it was found that the Learner had learned a very specific rule regarding the reduction of preposition/subordinate-conjunction combinations late in the learning process. 'The learned rule was:

PREVIAA $=N:$ P|SUBCONJ $\rightarrow$ SUBCONJ

Table 5: Ambiguously tagged texts, Complex Verbs

\begin{tabular}{|l|c|c|}
\hline Set & \# of rules learned & Score \\
${ } }$ & 125 & $81.7 \%$ \\
Srrall & 212 & $89.6 \%$ \\
Medium & 323 & $90.3 \%$ \\
Full & 564 & $90.2 \%$ \\
\hline (none) & 0 & $78.0 \%$ \\
\hline
\end{tabular}

'This rule was learned late in the learning process when most P/SUBCON.J pairs had alroady been reduced. However, as one can see from the context of the rule, it will apply in a large number of cases in a text. The Rule Tagger notes this and applies the rule early, thus incorrectly changing many I'SUBCONJ pairs to SUBCONJ and reducing the accuracy of the tagging. Since this phenomenon never occurred in any of the other learning rums, onc can see that the learning process can be heavily influenced by the choice of input texts.

\subsection{Effect of Rule Application Parameters}

'The next tests performed involved using rules generated above and changing parameters to the Rule 'Tagger to sec how the scores would be influenced. In the following test, we used the simple verb tag set rules but varied the r-tagfredom parameter and the seq parameter. 'The results can be found in Table 6.

Table 6: Ambiguously tagged texts, Simple Verbs

\begin{tabular}{|l|l|l|l|}
\hline Set & $\begin{array}{l}\text { R-'ag- } \\
\text { frectom }\end{array}$ & $\begin{array}{l}\text { Score } \\
\text { (best-rule- } \\
\text { first) }\end{array}$ & $\begin{array}{l}\text { Score } \\
\text { (learned- } \\
\text { sequence) }\end{array}$ \\
\hline 'T'iny & 1 & $82.7 \%$ & $80.2 \%$ \\
& 2 & $82.5 \%$ & $80.6 \%$ \\
& 3 & $82.1 \%$ & $80.5 \%$ \\
\hline Small & 4 & $81.9 \%$ & $80.5 \%$ \\
\hline & 2 & $90.1 \%$ & $89.8 \%$ \\
& 3 & $91.5 \%$ & $89.9 \%$ \\
& 4 & $91.5 \%$ & $89.9 \%$ \\
& 1 & $91.5 \%$ & $89.9 \%$ \\
\hline Medium & 1 & $90.5 \%$ & $90.6 \%$ \\
& 2 & $91.8 \%$ & $90.5 \%$ \\
& 3 & $91.8 \%$ & $90.5 \%$ \\
& 4 & $91.8 \%$ & $90.5 \%$ \\
\hline & 1 & $82.1 \%$ & $79.8 \%$ \\
& 2 & $83.0 \%$ & $80.0 \%$ \\
& 3 & $81.7 \%$ & $80.0 \%$ \\
& 4 & $81.5 \%$ & $80.0 \%$ \\
\hline
\end{tabular}

Although the variations are slight, the best value for the r-tagfreedom parameter seems to be at an ambiguity level of 2 . It scems that the strategy of reducing the ambiguity as quickly as possible (best-rulc-first) is better than following the ordering of the rules by the Learner. This may well be due to the fact that the ordering of the rules as produced by the Learner is dependent on the training texts. Since the test set was a different set of texts, the ordering of the rules was not as applicable to them as to the training texts, and so the tagging performance suffered. 


\subsection{Effect of Hand-tagged Texts}

After oxamining the results from the aloove experiments, we realized that some of the closed-class words in Spanish are almost always ambiguons (c.g., prepositions are usually ambiguous between PREP and SUBCONJ, and determiners between D) lel' and P'R,O). 'This means that the learner will never learn a rule to disambiguate those closedclass cases because there will rarely be mambiguous contexts in the training lexts tagged by the Initial State Annotator. 'That is, mnlikeopen-class words, we will not find new nuanubiguous closedchass words in texts precisely becallse thore is only a closed set of them. 'Thus, we decided to introduce a small number of hand-tagged texts into the training set given to the Learuer. Since the handlagged tiexts have "correct" examples of viulous phenomena, the learner should bo able to find good examples in them to learn from.

for our tests, we defined four sels of haundlagged texts that we added to the "Simall" (3066 words) set of ambiguousty lagged texts. 'Iho brakdown is in Table 7.

Tablo 7: Iland-tagged 'lraining sols

\begin{tabular}{|l|l|}
\hline Set & Words \\
\hline Sinall & 218 words \\
Medium & 588 words \\
Large & 906 words \\
Pull & 1791 words \\
\hline
\end{tabular}

Again, the learner was set to have a tight restriction on using context for learning (freedom I) and a loose restriction on context lor applying the learned rules (tagfreedom 10). 'The Rule 'Tagger was given a moderately-tight restriction on using context for reduction rule application (fredom 2 ). 'The best-rule-first mode of the Rule: 'Tagger was used.

The results, as shown in 'lable 8 , are slightly better than when using only ambiguously tagged texts. It is interesting to note that the higher accuracy was achieved with fewer rules. In fact, all experiments resulted in learning a litule over 200) rules.

'lable 8: Ambiguous/Unambiguous 'lexts, Simuple Vorbs

\begin{tabular}{|l|c|c|}
\hline Sot & \# of rules learmed & Score \\
\hline Small & 210 & $91.2 \%$ \\
Mediun & 211 & $92.1 \%$ \\
Large & 205 & $92.1 \%$ \\
rull & 202 & $92.1 \%$ \\
\hline (nonc) & 211 & $91.5 \%$ \\
\hline
\end{tabular}

In addition to the experimentis above, we wanted to know if the introduction of hand- lagged textis into the "lull" anbiguously tagged set would improve its rather low seore (cl. 'latble 4). We performed an experiment using simple verb tags, the "Full" ambignously tagged texts, and the "linll" hand-tagged texts. The results were 122 rules learned with a score of $92.1 \%$, which tied with the "Snall" ambiguously tagged set for" achieving the highest accuracy of all of the learning/tagging runs, a full $13.5 \%$ higher than using no learning.

\section{Problems and Possible Improvements}

Althongh our Spanish POS tagger performed reasonably well, achicving an improvenent of $13.5 \%$ in accuracy over randomly picking tags, there were several problems that prevented the system from reachiug an even higher score.

\subsection{Loaning Problem}

As discussed in Snction 3.3, anbiguous closed. class words (c.g., prepositions, determiners, etc.) cannot be reduced when there are no unambiguras examples of them in the tratomg texts. 'This is prevalent, in Spanish, where most prepositions an also be subordinate conjunctions, deteminers can be pronoms, ete. $\Lambda$ fow hand-tagged textis are required lo karn good rules for reducing the ambignity on these words. It is possible, however, that such texts can be disambiguated only for their always ambiguous closed-class words but not nuambiguous closed-class words or open-class words. Such an experinent similar to setective sampling discussed in I)agan and Engelson (1)agan and lingelson, 1995) would be useful in the fulure because, il' it is true, it will reduce the cost ol mantlal hageing considerably.

\subsection{Lexicon Problem}

Problems that becance apparent as we ran more lests were the incompleteness and mistakes in the lexicon. While the lexicon, derived from the Collins Spanish-Linglish dictionary, was quite rich in words, its tag set did not always match the tag defintions we omployed. for example, our tag set. distinguishes proper nomms (PROPN) and nomus $(N)$, whereas the ('ollins dietionary marked both as nomus (N). We have added our existing proper mame lists to the lexicon to partially solve this problem, but the lists are currently limited to bocalion nantes and poople's first namess.

We also found several mistakes in the collins definitions (e.g., several adverbs ending "-tuente" were classified alljectives). Nlthough we lixed these mistakes as we noticed therr, it is diflicult. to know how many such errors still remain in the Iexicon.

It turned out that the ineompleteness of the lexicon was another fundanental problem to Brill's mosupervised leming algorithm. 'That is, when 
the lexicon does not list all the possible tags for a word, the tagger is very likely to make a mistake. This is because the learner is trained to reduce the ambiguity of possible tags of a word (say $\mathrm{N}$, $V, \Lambda D J$ tags), but if the lexicon lists only a subsct of the possible tags (say $\mathrm{N}$ and $\mathrm{V}$ tags), the system will never learn to assign an ADJ tag even when the word is used as an adjective.

This type of problem was observed frequently when words are ambiguous between proper nouns and some other parts-of-speech such as "Flores (ADJ/PROPN)," "Lozano (ADJ/PROPN)," "van (V/PROPN)",3, "Scrra (V/PROPN)," etc. because not all the proper nouns are in the lexicon.

The problems described above did not occur in Brill's experiments because he derived the lexicon from a POS-tagged corpus and used the untagged version of the same corpus for training and testing. Thus, he used an "optimal" lexicon which contains all the words with only parts-of-speech which appeared in the corpus. In addition, in such a corpus, rarely used POS tags of a word are less likely to occur, and words are less likely to be ambiguous. 'Thus, in a sense, his "unsupervised learning" experiments did take advantage of a large POS-tagged corpus.

\section{Related Works}

It is very difficult to compare performances be tween taggers when accuracy depends on quality of corpora and lexicons, and maybe on characteristics of languages. But in this section, we compare our tagger with Hidden Markov Model-based taggers.

$\Lambda$ more widely used algorithm for unsupervised learning of a POS tagger is Hidden Markov Model (IIMM). Cutting el al. (Cutting et al., 1992) and Melialdo (Merialdo, 1994) used II MM to learn Fnglish POS taggers while Chanod and 'Tapanainen (Chanod and Tapanainen, 1995), Feldweg (Feldweg, 1995), and León and Serrano (León and Serrano, 1995) ported the Xerox tagger (Cutting et al., 1992) to French, German, and Spanish respectively. One of the drawbacks of an HMM-based approach is that laborious manwal tuning of symbol and transition biases is necessary to achieve high accuracy. Without tuned biases, the German Xerox tagger achieved $85.89 \%$ while the French Xerox tagger achieved $87 \%$ accuracy. After one man-month of tuning biases, the accuracy of the French tagger increased to $96.8 \%$. One could derive such biases from a corpus, as discussed in (Merialdo, 1994), but it unfortunately requires a tagged corpus.

'The best accuracy of the Spanish Xerox tag'ger was $91.51 \%$ for the reduced tag set (174 tags)

\footnotetext{
${ }^{3}$ It can be a part of a last nanne as in "van Mahler", but also is an inflected form of "ir".
}

with the base accuracy (i.e. no training) of $88.98 \%$ while the best accuracy of our tagger is currently $92.1 \%$ for the simple tag set (39 tags) with the base accuracy of $78.6 \%$. 'The lower base accuracy in our experiment is probably due to the large number of entries in the Collins dictionary.

\section{Summary}

Our Spanish Part of Speech 'T'agger is a successful implementation and extension of Brill's unsupervised lcarning algorithm that reduces the ambiguity of part-of-speech tags on words in Spanish texts.

The system requires few, if any, hand-tagged texts to bootstrap itself. Rather, it merely requires a Spanish lexicon and morphological analyzer that can tag words with all their possible parts-of-speech. Given that the system performs at approximately $92 \%$ accuracy even with the aforementioned problems and with the inclusion of unknown words, we would expect that this system could achieve better results, approaching those of similar English-language POS taggers, when these problems arc rectified.

\section{References}

Eric Brill. 1995. Unupervised learning of disambiguation rules for part of speech tagging. In Procecdings of the 3rd Workshop on Very Large. Corpora.

Jean-Pierre Chanod and Pasi lapanainen. 1995. Tagging French - Comparing statistical and a constraint-based method. In Proceedings of the HACL-95.

D. Culting, J. Kupiec, J. Pedersen, and P. Sibun. 1992. A Practical Part-of-Speech Tagger. In Proccedings of the Third Conference on Applicd Natural Language Processing.

Ido Dagan and Scan P. Bngelson. 1995. Selective Sampling in Natural Language Learning. In Proceedings of the IJCAI Workshop on New Approach to Lcarning for Nalural Language Processing.

Nelmut Feldweg. 1995. Implementation and lival. uation of a (xerman IIMM for POS Disambiguation. In Proceedings of the EACL, SICI)AT Workshop.

Fernando Sánchez León and Amalio P. Nieto Serrano. 1995. Development of a spanish version of the xerox tagger. In Procedings of the $X I$ Congreso de la Sociedad lispañola para el Proce. samiento del Lenguaje Nalural (SWPLN'95).

Bernard Merialdo. 1994. Tagging English Text with a Probabilistic Model. Computalional Linguistics, $20(2)$. 